\title{
Spectral and quantum chemical study of tautomeric and ion transformations of 2-(2-(2-hydroxy-5-sulfamoylphenyl)- hydrazono)-3-oxo- $N$-phenylbutanamide
}

\author{
(C) Anh Thi Ngoc Vu, ${ }^{1,2+}$ Mikhail A. Ryabov, ${ }^{1}$ \\ Olga V. Kovalchukova, ${ }^{1,3 *}$ and Dmitry S. Gusarov ${ }^{3}$ \\ ${ }^{1}$ Department of General Chemistry. Peoples' Friendship University of Russia (RUND University). Miklukho- \\ Maklaya St., 6. Moscow, 117198. Russia. Phone: +7 977879 0668. E-mail: vuanh0000@gmail.com \\ ${ }^{2}$ Department of Pharmaceuticals. Buon Ma Thuot University. Ha Huy Tap St., 298. \\ Buon Ma Thuot. Daclak, 630000. Vietnam. \\ ${ }^{3}$ Department of Organic Chemistry. A.N. Kosygin Russian State University (Technology. Design. Art). \\ Sadovnicheskaya St., 33. P.1. Moscow, 115035. Russia.
}

*Supervising author; ${ }^{+}$Corresponding author

Keywords: quantum chemical calculations, 2-arylhydrazono-1,3-dicarbonyl compounds, tautomeric, conformer and ionic forms.

\begin{abstract}
In this paper, using the method DFT/B3LYP of quantum chemical modeling, the stability of tautomeric, conformer, and anionic forms of 2-(2-(2-hydroxy-5-sulfamoylphenyl) hydrazone-3-oxo- $N$-phenylbutanamide $\left(\mathrm{H}_{2} \mathrm{~L}\right)$ was studied and the geometric, electronic structure and bond lengths of molecules in various tautomeric forms were determined. It was shown that the stability of tautomeric molecules $\mathrm{H}_{2} \mathrm{~L}$ as anionic forms was determined by the number of existing intermolecular hydrogen bonds (closing six-membered and fivemembered cycles). The most stable tautomeric and conformal forms of an organic molecule and its dianion are proposed. The interatomic distances, angles, charges on atoms, IR and electronic absorption spectra of tautomeric forms of the molecule and dianion $\left(\mathrm{H}_{2} \mathrm{~L}\right.$ and $\left.\mathrm{L}^{2-}\right)$ were calculated. In this work, we studied the electronic absorption spectra in an aqueous-alcoholic solution and found that in an alkaline medium the molecule transforms into the ionic form with a bathochromic shift of $47 \mathrm{~nm}$, corresponding to the results of the shift of the long-wavelength band electronic absorption spectra upon transition from the hydrazotautomer (form a) to the dianion form (form b") at $92 \mathrm{~nm}$ obtained by TD-DFT. The calculated results for the distance and angles between atoms are in good agreement with the experimental data of the molecule by X-ray diffraction analysis. It was shown that the IR spectrum of the stable form appears two absorption bands in the region of 1690 and $1655 \mathrm{~cm}^{-1}$, which are attributed to stretching vibrations of carbonyl groups $(\mathrm{C}=\mathrm{O})$, bound by a strong intramolecular hydrogen bond and the presence of the NH-hydrazone fragment of the ${ }^{1} \mathrm{H}$ NMR spectrum confirmed the most stable for a neutral molecule is the hydrazone form.
\end{abstract}

\section{References}

[1] E.V. Shchegol'kov, Ya.V. Burgart, O.G. Khudina, V.I. Saloutin, O.N. Chupakhin. 2-(Het) arylhydrazono1,3-dicarbonyl compounds in organic synthesis. Russian Chemical Reviews. 2010. Vol.79. No.31. P.3161. (russian)

[2] K.I. Pashkevich, V.I. Saloutin. Fluorine-containing $\beta$-Ketoesters. Russian Chemical Reviews. 1985. Vol.54. P.1185. (russian)

[3] H. Zollinger. Color Chemistry: Syntheses, Properties, and Applications of Organic Dyes and Pigments. Wiley-VCH, Weinheim. 2003.

[4] H. Zollinger. Diazo Chemistry I: Aromatic and Heteroaromatic Compounds. Wiley, New York. 1994.

[5] D.S. Gusarov, M.A. Ryabov, Thi Ngok Anh Vu, and O.V. Kovalchukova. Quantum-chemical modeling of structure and properties of nitrogen derivatives of phloroglucinol. Butlerov Communications. 2019. Vol.57. No.3. P.51-57. DOI: 10.37952/ROI-jbc-01/19-57-3-51

[6] O.V. Kovalchukova, S.B. Strashnova, P.V. Strashnov, E.P. Romashkina, O.V. Volyanskiy, and K.I. Kobrakov. Spectral and quantum-chemical studies of tautomeric and ionic transformations of azo-dyes based upon methylfloroglucine. Butlerov Communications. 2011. Vol.24. No.1. C.90-94. ROI: jbc-01/11$24-1-90$ 
SPECTRAL AND QUANTUM CHEMICAL STUDY OF TAUTOMERIC AND ION TRANSFORMATIONS... $106-113$

[7] S.B. Strashinova, O.V. Kovalchukova, E.P. Romashkina, O.V. Avramenko, and O.V. Volyansky. Quantum-chemical modeling of the metal chelate cycles of methylphloroglucine phenylazo derivatives. Butlerov Communications. 2013. Vol.33. No.1. P.78-82. ROI: jbc-02/13-33-1-78

[8] A.D. Becke, Density-functional thermochemistry. III. The role of exact exchange. J. Chem. Phys. 1993. Vol.98. P.5648-5652.

[9] C. Lee, W. Yang, and R.G. Parr Development of the Colle-Salvetti correlation-energy formula into a functional of the electron density. Phys. Rev. 1988. B37. P.785-789.

[10] P.J. Stephens, F.J. Devlin, C.F. Chabalowski, M.J. Frisch. Ab initio calculation of vibrational absorption and circular dichroism spectra using density functional force fields. J. Phys. Chem. 1994. Vol.98. P.11623-11627.

[11] A. Schaefer, C. Huber, R. Ahlrichs. Fully optimized contracted Gaussian-basis sets of triple zeta valence quality for atoms Li to Kr. J. Chem. Phys. 1994. Vol.100. P.5829-5835.

[12] Alex A. Granovsky. Firefly version 7.1.G, www http://classic.chem.msu.su/gran/firefly/index.html 